\title{
Multi-objective Optimization of a Transportation Network of a HMSC
}

\author{
Ahmed Mohammed and Qian Wang \\ School of Engineering, Faculty of Technology, University of Portsmouth, Portsmouth, UK
}

\begin{abstract}
In this paper, we investigated a transportation network of a tri-layer Halal meat supply chain (HMSC) in which Halal meat transportation process was monitored by a Radio Frequency Identification (RFID) communication system to ensure safety and integrity of Halal meats. This monitoring system is subject to an extra cost in investment that needs to be taken into account. Thus, a multi-objective linear programming model (MOLPM) was developed aiming to minimize the total cost in transportation and number of transportation vehicles and maximize the service level in product quantity as requested by abattoirs and retailers. The facility location-allocation problem in farms, abattoirs and retailers needs also to be addressed in relevance to the quantity flow of products from farms to abattoirs and from abattoirs to retailers. The utility function method was employed to obtain Pareto-optimal solutions and the global criterion method was used for searching the most suitable Pareto solution by minimizing the distance to its ideal objective value. The research work shows that the developed model can be useful for supply chains design through a case study based on numerical results.
\end{abstract}

\section{Introduction}

A food supply chain network includes food production, distribution and consumption. Design of a supply chain network involves strategic decisions in allocating facilities that should be established and tactical decisions in terms of product flows throughout the supply chain network. To maintain a competitive share in the market, it requires an effective and efficient supply chain network by minimizing costs and maximizing profits. For Muslim people, Halal food accounts for over sixteen percent of the global food trade. Halal is an Arabic word which means lawful or permitted according to the Islamic law. Integrity of Halal meat products refers to a Halal meat production and distribution which must comply with the Islamic Shari'ah (laws). This includes processes of livestock feeding, slaughtering, packing, storing and transporting in a Halal meat supply chain (HMSC) network.

Based on the reviewed literature, few research works applied multi-objective optimization models in the context of food supply chain networks. Vanek and Sun [1] suggested an energy minimizing model that considers both the shipment and the perishable produce production. These Authors proposed to ship an additional produce quantity to guarantee the consumer demand satisfaction, due to the produce deterioration. Bosona and Gebresenbet [2] investigated the existing flow of food products from producers to consumers. To this aim, an integrated logistics network embracing producers, retailers, a collection center and a distribution center was proposed.
Rong et al. [3] developed a mixed integer linear programming model for a fresh food supply chain aimed at guaranteeing the produce quality for consumers. Sahar et al. [4] proposed a multi-objective optimization model for a two-layer dairy supply chain. The model aimed at minimizing $\mathrm{CO}_{2}$ emissions from transportation and total costs in the distribution chain.

Furthermore, different researches exist in the literature regarding the application of multi-objective optimization models into different supply chain network design and distribution problems. Revelle and Laporte [5] addressed supply chain design problems, these problems may be solved by a number of compromising solutions known as Pareto solutions. Sabri and Beamon [6] developed a multi-objective programming model to obtain an optimum supply chain network performance by considering two conflicting objectives including minimization of the total cost and maximization of volume flexibility of plants. Chan et al. [7] presented a hybrid-genetic algorithm to solve the distribution problem of a supply chain network. Three objectives were optimized including total cost, completion lead time and capacity utilization. Chen and Lee [8] developed a multi-objective model for a multi-echelon supply chain network seeking a compromise among conflicting objectives including fair profit distribution, safe inventory levels, customer service levels, and uncertain demands of products. Altiparmak et al. [9] proposed a genetic algorithm focusing on minimization of inbound and outbound distribution costs and maximization of customer services in terms of delivery time and capacity 
for distribution centers. Schütz et al. [10] formulated a decision support system using a two-stage stochastic program with respect to minimizing costs of investment and operations of a supply chain. Shankar et al. [11] investigated a two-echelon supply chain for locating distribution centers with a minimal cost using the genetic algorithm, which was compared with using the Lagrangian heuristic approach.

This paper presents a development of a multiobjective linear programming model for a RFID-based HMSC proposed by Mohammed and Wang [12]. The model aimed at solving the facility location-allocation problem and the quantity flow of products considering three objective functions by minimizing the total transportation cost, number of transportation vehicles and maximizing the service level in satisfying the quantity of Halal meat products requested by abattoirs and retailers.

\section{The HMSC model}

Figure 1 illustrates a tri-layer Halal meat supply chain (HMSC), which consists of farms, abattoirs and retailers. The objectives of this model include: (1) minimization of the total transportation cost $\left(\mathrm{N}_{1}\right) ;(2)$ minimization of the number of required vehicles for transportation $\left(\mathrm{N}_{2}\right)$; and (3) maximization of service level $\left(\mathrm{N}_{3}\right)$. The model is also aimed at helping design the HMSC network in numbers and locations of farms and abattoirs in response to quantity of products flow from farms to abattoirs and from abattoirs to retailers.

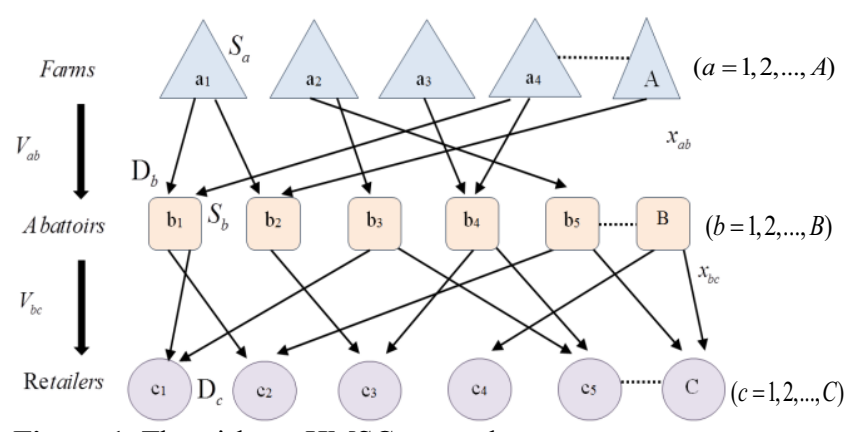

Figure 1. The tri-layer HMSC network.

The mathematical notations, parameters and decision variables are as follows:

\section{Indices}

$A$ index of farms $(a=1,2, \ldots, A)$

$B$ index of abattoirs $(b=1,2, \ldots, B)$

$C$ index of retailers $(c=1,2, \ldots, C)$

\section{Parameters}

$R_{a b}$ unit transportation cost (GBP) per mile from farm $a$ to abattoir $b$

$R_{b c}$ unit transportation cost (GBP) per mile from abattoir $b$ to retailer $c$

$T_{a b} \quad$ RFID tag cost (GBP) per item transported from farm $a$ to abattoir $b$

$T_{b c}$ RFID tag cost (GBP) per item transported from abattoir $b$ to retailer $c$
$S M_{a b}$ RFID system cost (GBP) required per lorry travelling from farm $a$ to abattoir $b$

$S M_{b c}$ RFID system cost (GBP) required per lorry travelling from abattoir $b$ to retailer $c$

C transportation capacity (units) per lorry

$S_{a}$ maximum supply capacity (units) of farm $a$

$S_{b}$ maximum supply capacity (units) of abattoir $b$

$D_{b}$ minimum demand (in units) of abattoir $b$

$D_{c}$ minimum demand (in units) of retailer $c$

\section{Variables}

$x_{a b}$ quantity of livestock transported from farm $a$ to abattoir $c$

$x_{b c}$ quantity of processed meats transported from abattoir $b$ to retailer $c$

$V_{a b}$ number of expected required vehicles to transport livestock from farm $a$ to abattoir $b$

$V_{b c}$ number of expected required vehicles to transport processed meats from abattoir $b$ to retailer $c$

$$
\begin{aligned}
& y_{a}=\left\{\begin{array}{l}
1: \text { if farm } a \text { is opened } \\
0: \text { otherwise }
\end{array}\right. \\
& y_{b}=\left\{\begin{array}{l}
1: \text { if abattoir } b \text { is opened } \\
0: \text { otherwise }
\end{array}\right.
\end{aligned}
$$

Thus, by minimizing the total transportation cost $\left(\mathrm{N}_{1}\right)$, number of transportation vehicle $\left(\mathrm{N}_{2}\right)$ and maximizing service level $\left(\mathrm{N}_{3}\right)$, the model formulation is given as follows:

$$
\begin{gathered}
\operatorname{Min} N_{1}=\sum_{a} \sum_{b} T_{a b} y_{a}+\sum_{b} \sum_{c} T_{b c} y_{b} \\
+\sum_{a} \sum_{b} S M_{a b} V_{a b}+\sum_{b} \sum_{c} S M_{b c} V_{b c} \sum_{a} \sum_{b} R_{a b} x_{a b}+\sum_{b} \sum_{c} T_{b c} x_{b c} \\
\operatorname{Min} N_{2}=\sum_{a} \sum_{b} R_{a b} V_{a b}+\sum_{b} \sum_{c} R_{b c} V_{b c} \\
\max N_{3}=\frac{\left[\left(\frac{\sum_{a} \sum_{b} x_{a b}}{\sum_{b} D_{b}}\right) y_{a}+\left(\frac{\sum_{b} \sum_{c} x_{b c}}{\sum_{c} D_{c}}\right) y_{b}\right]}{2}
\end{gathered}
$$

s.t.

$$
\begin{gathered}
\sum_{a} x_{a b} \leq S_{a} y_{a} \quad \forall b \in B \\
\sum_{b} x_{b c} \leq S_{b} y_{b} \quad \forall c \in C \\
\sum_{a} x_{a b} \geq D_{b} \quad \forall b \in B \\
\sum_{b} x_{b c} \geq D_{c} \quad \forall c \in C \\
D_{b} \geq \sum_{c} x_{b c} \quad \forall b \in B \\
\sum_{e} V_{a b}=x_{a b} / \mathrm{C}_{l} \quad \forall b \in B
\end{gathered}
$$




$$
\begin{gathered}
\sum_{b} V_{b c}=x_{b c} / \mathrm{C}_{l} \quad \forall c \in C \\
V_{a b}, V_{b c} \text { integer } \\
x_{a b}, x_{b c} \geq 0 \quad \forall a, b \\
y_{a}, y_{b} \in\{1,0\}, \quad \forall a, b
\end{gathered}
$$

Equations 4 and 5 are constraints in capacity at farms and abattoirs, respectively. Equations 6, 7 and 8 determine the demands of abattoirs and retailers. Constraints 9 and 10 provide the estimated number of vehicles indicated in the objective function 2 . Constraints 11,12 and 13 prohibit non-negativity and the non-binary restrictions on decision variables.

\subsection{Solution methodology}

To obtain Pareto solutions, the utility function method was used and the most suitable Pareto solution was selected using the global criterion method. The utility of each Pareto solution is determined by a sum of the scaled objective functions. The scalar value $\lambda$ for each objective is determined by decision makers according to the importance of each objective [13]. In this work, the objective function (or utility function) $U$ is expressed as follows:

$$
U\left(N_{1}, N_{2}, N_{3}\right)=\left\{\sum_{i=1}^{3} \lambda_{i} N_{i} \mid \lambda_{i}>1, \sum_{i=1}^{3} \lambda_{i}=1\right\}
$$

The next step after obtaining the Pareto-optimal solutions is to find the best trade-off solution. From the decision maker's view-point, choosing a solution of Pareto-optimal solutions is called a posteriori method [14]. There are several methods for selecting the most suitable solution in a multi-objective problem. In this case, the global criterion method was used in this study for determining the best solution by minimizing the distance to the ideal objective value $\left(N_{i}^{*}\right)$ [15], it can be given by:

$$
\min \mathrm{N}=\left(\sum_{i=1}^{3}\left|N_{i}-N_{i}^{*}\right|^{\rho}\right)^{1 / \rho} ; 1 \leq \rho \leq \infty
$$

Figure 2 shows the block diagram of the MOLPM development.

\section{Implementation and evaluation}

A case study was used for examining the validity of the developed mathematical model. Table 1 shows data collected from the Halal Meat Committee in the UK [16]. Figure 3 illustrates the locations of four farms, five abattoirs and eleven retailers in a region. The computational solutions and their optimization were conducted using LINGO. First, each objective function (OF) was individually optimized to obtain Table 2.

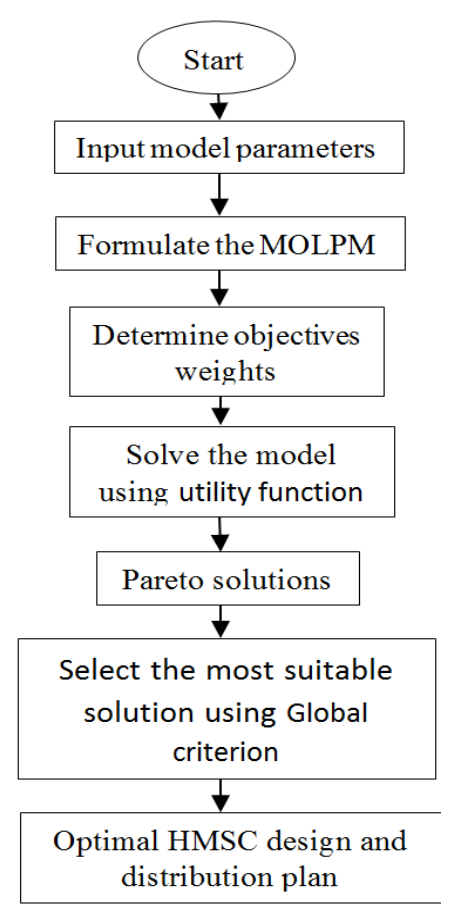

Figure 2. Block diagram of the MOLPM development.

Table 1. Collected HMC data.

\begin{tabular}{lll}
\hline$A=4$ & $R_{b c}=15-20$ & $\mathrm{D}_{B}=600-1.5 \mathrm{~K}$ \\
$B=5$ & $S_{a}=1.2 \mathrm{~K}-2.5 \mathrm{~K}$ & $\mathrm{D}_{C} ?=100-200$ \\
$C=11$ & $S_{b}=1 \mathrm{~K}-1.8 \mathrm{~K}$ & $S M_{b c}=800$ \\
$R_{a b}=15-20$ & $C_{l}=20-30$ & $T_{a b}=0.15$ \\
$T_{a b}=0.15$ & $S M_{a b}=800$ & \\
\hline
\end{tabular}

The optimal boldface value for each OF was used as an ideal value for the decision making method (described in sub-section 2.1). In order to optimize the three objective functions and obtain Pareto solutions, ten different scalar values $\left(\lambda_{i}\right)$ were assigned. Table 3 shows the obtained seven solutions of the Pareto frontier. It is noticed that the utility function method has a disadvantage as it only obtains seven rather than ten results

Table 2. Computational results with solutions provided based on each objective function to be minimized $\left(N_{1}, N_{2}\right)$ and maximized $\left(N_{3}\right)$ individually.

\begin{tabular}{cccc}
\hline $\begin{array}{c}\text { Objective } \\
\text { functions }\end{array}$ & $\min N_{1}$ & $\min N_{2}$ & $\max N_{3}$ \\
\hline$O F_{1}$ & $\mathbf{5 5 4 5 5}$ & 56384 & 187673 \\
$O F_{2}$ & 26 & $\mathbf{2 5}$ & 73 \\
$O F_{3}$ & 0.899 & 0.895 & $\mathbf{0 . 9 9 9}$ \\
\hline
\end{tabular}

when the scalar value of the first objective function was set to be less than 0.4 (solutions 8,9 and 10). This may limit decision makers for measuring the importance of the three objectives. Table 3 also shows that the objective functions are conflicted as it is impossible to achieve optimal values for all the three objectives at a time. This conflict among the three objective functions is further illustrated in Figure 4. It is noteworthy in Table 3 that a 
number of facilities were eliminated. For instance, solution 3 was obtained with the weight $(0.8,0.1,0.1)$, the results only gave one farm (located in Yorkshire). This farm can supply the required livestock to three abattoirs located in York, Bradford and Sheffield with respect to the demands of the eleven retailers. When designing the transportation network of a HMSC, decision makers need to determine the best solution based on a number of alternative possibilities. In this study, the global criterion method was used to select the most suitable solution. Accordingly, if $\rho$ is set to 1 for equation
15 , solution 4 is the most suitable solution as it gives the minimum distance to the ideal objective value. This solution has a transportation cost of 84,569 GBP with 48 vehicles and a service level of $93.3 \%$. This solution requires an establishment of three farms to supply livestock to three abattoirs. Table 4 shows the quantity of products flow between farms $(1,2,3)$ and abattoirs $(1,2$, 4 ) and eleven retailers. For instance, u2, 1 implies that farm 2 is demanded to supply 800 livestock to abattoir 1 , and v1, 1 implies that abattoir 1 is demanded to supply 850 packages of processed meats to retailer 1 .

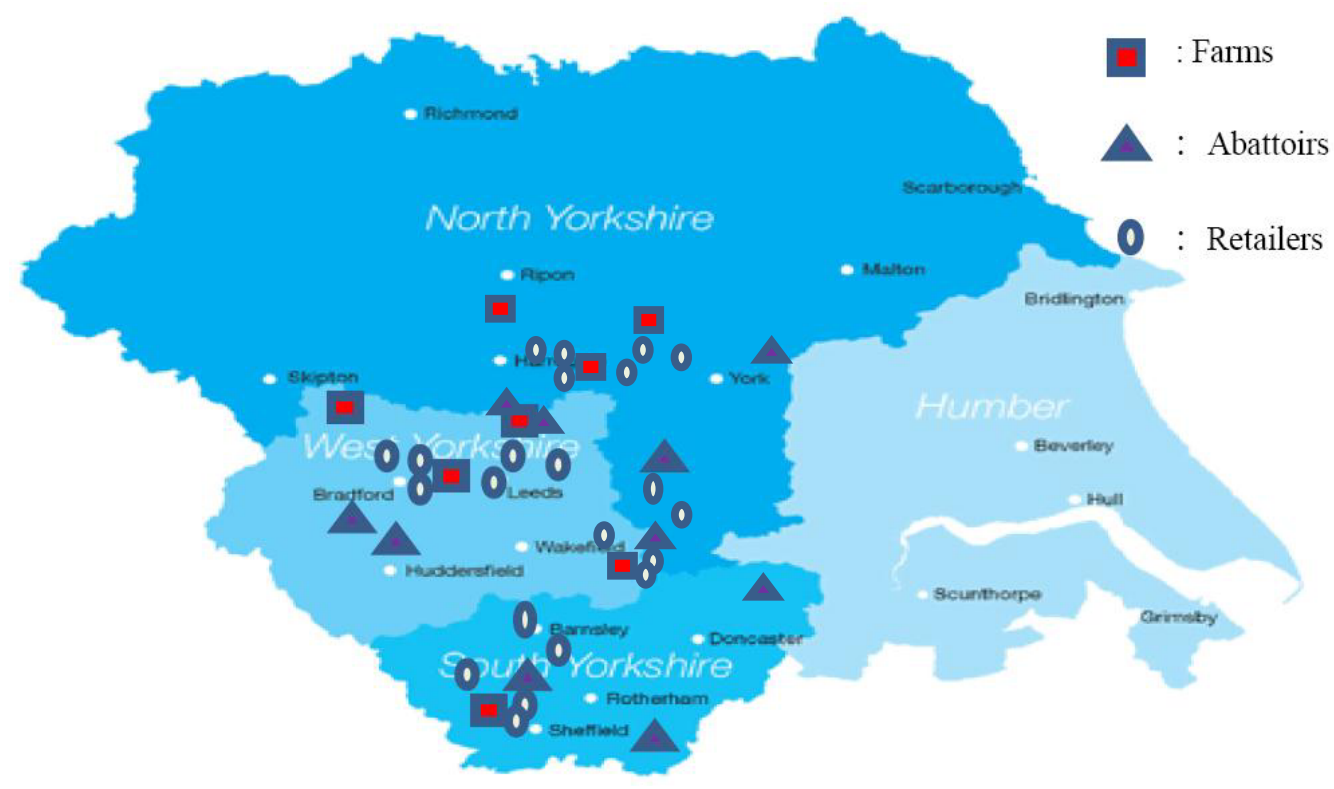

Figure 3. The HMSC network.

Table 4. The quantity of products flow of the HMSC.

\begin{tabular}{llll}
\hline & Quantity & Facilities & Quantity \\
\hline $\mathrm{u}_{1,4}$ & 1200 & $\mathrm{v}_{1,1}$ & 850 \\
$\mathrm{u}_{2,1}$ & 800 & $\mathrm{v}_{1,3}$ & 210 \\
$\mathrm{u}_{2,4}$ & 1200 & $\mathrm{v}_{1,6}$ & 690 \\
$\mathrm{u}_{3,1}$ & 1000 & $\mathrm{v}_{2,5}$ & 290 \\
$\mathrm{u}_{3,2}$ & 290 & $\mathrm{v}_{2,10}$ & 100 \\
$\mathrm{v}_{2,11}$ & 700 & $\mathrm{v}_{2,8}$ & 160 \\
$\mathrm{v}_{4,6}$ & 850 & $\mathrm{v}_{2,9}$ & 110 \\
$\mathrm{v}_{4,7}$ & 450 & $\mathrm{v}_{2,2}$ & 350 \\
$\mathrm{v}_{2,4}$ & 220 & & \\
\hline
\end{tabular}

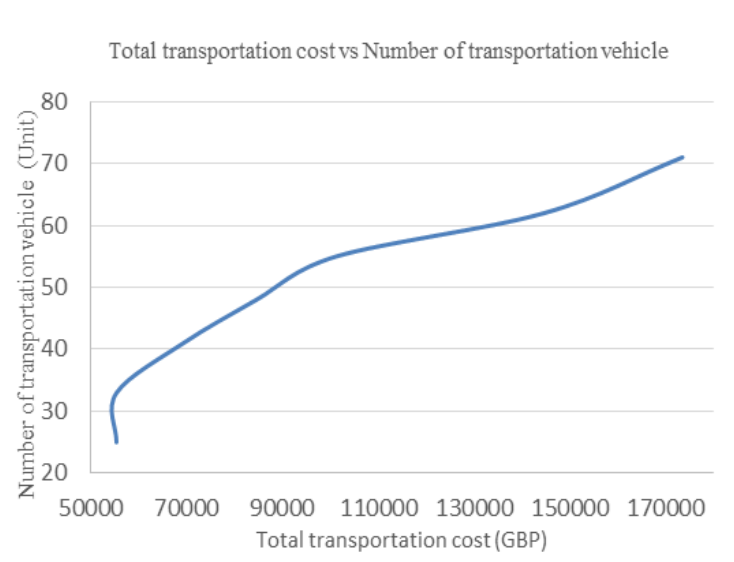

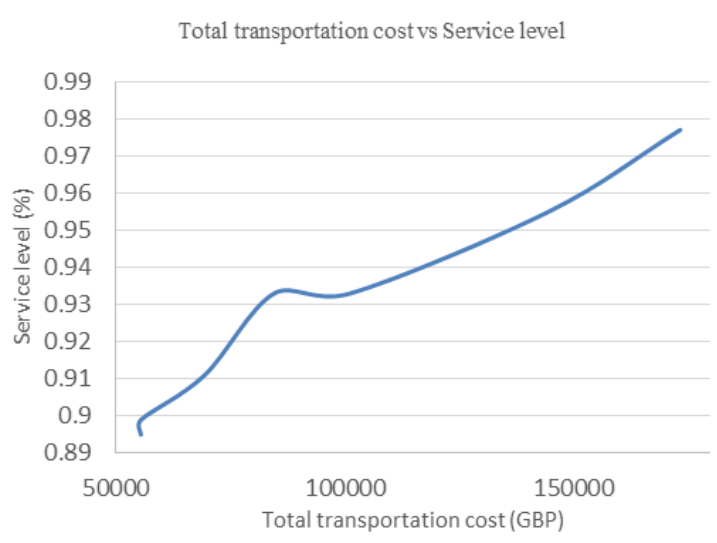

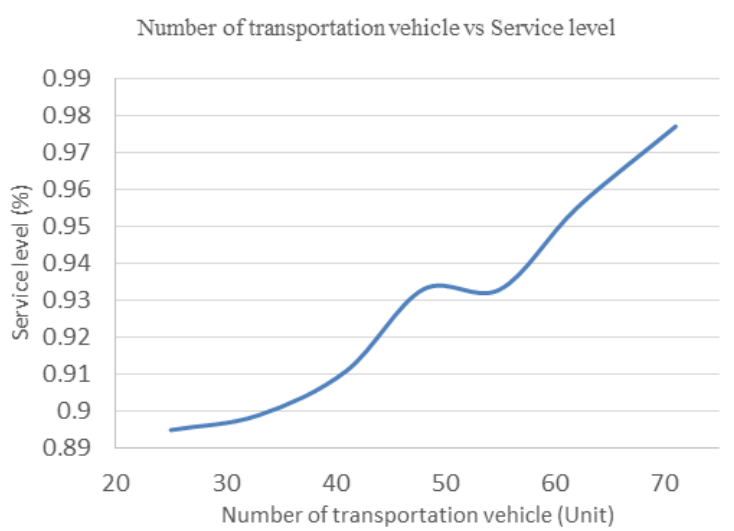

Figure 4. The confliction in values related to $\mathrm{OF}_{1}, \mathrm{OF}_{2}$ and $\mathrm{OF}_{3}$. 
Table 3. Pareto solutions for the HMSC aiming to three objective functions.

\begin{tabular}{|c|c|c|c|c|c|c|}
\hline \multirow{2}{*}{\multicolumn{2}{|c|}{$\frac{\text { Objectives weights }}{\left(\lambda_{1}, \lambda_{2}, \lambda_{3}\right)}$}} & \multicolumn{3}{|c|}{ Objectives values } & \multicolumn{2}{|c|}{ Open facilities } \\
\hline & & $\begin{array}{c}\operatorname{Min} \mathrm{OF}_{1} \\
(\mathrm{GBP})\end{array}$ & $\begin{array}{c}\text { Min } \mathrm{OF}_{2} \\
\text { (Units) }\end{array}$ & $\begin{array}{c}\operatorname{Max} \mathrm{OF}_{3} \\
(\%)\end{array}$ & Farms & Abattoirs \\
\hline 1 & $1,0,0$ & 55400 & 25 & 0.895 & 1,2 & 4,5 \\
\hline 3 & $0.8,0.1,0.1$ & 69343 & 41 & 0.911 & 1 & $3,4,5$ \\
\hline 4 & $0.7,0.15,0.15$ & 84569 & 48 & 0.933 & $1,2,3$ & $1,2,4$ \\
\hline 5 & $0.6,0.2,0.2$ & 101234 & 55 & 0.933 & $1,3,4$ & $1,2,3,4,5$ \\
\hline 8 & $0.3,0.35,0.35$ & - & - & - & - & - \\
\hline 9 & $0.2,0.4,0.4$ & - & - & - & - & - \\
\hline 10 & $0.1,0.45,0.45$ & - & - & - & - & - \\
\hline
\end{tabular}

\section{Conclusions}

This paper presented a development of a multi-objective mathematical model used for obtaining a compromise solution by optimizing three objectives simultaneously, which include (1) minimization of the total transportation cost, (2) minimization of the number of vehicles required for transportation and (3) maximization of the service level by satisfying all demands in quantity of meat products as requested by abattoirs and retailers. The model solves the facility location-allocation as well as the optimal quantity of product flows. The computed results showed that there were no feasible solutions when the scalar value for the first objective was set less than 0.4. A case study was used for verifying the developed mathematical model and the performance of the proposed solution methodology. Research findings indicate that the developed model is applicable and can be used as a reference for food supply chain designers. The model can be developed further as a fuzzy multi-objective model incorporating the uncertainty of input data such as varying demands and costs.

\section{Acknowledgment}

The authors would like to express their gratitude to the Higher Committee for Education Development (HCED) in Iraq for the financial support in this study.

\section{References}

1. F. Vanek, Y. Sun, Transport versus perishability in life cycle energy consumption: a case study of the temperature-controlled food product supply chain, Transp. Res. Part D Transp. Environ., 13, 383-391 (2009)

2. T. Bosona, G. Gebresenbet, I. Nordmark, D. Ljungberg, Integrated logistics Network for the supply chain of locally produced food, part I: location and route optimization analyses, J. Serv. Sci. Manag, 4, 174-183 (2011)

3. A. Rong, R. Akkerman, M. Grunow, An optimization approach for managing fresh food quality throughout the supply chain, Int. J. Prod. Econ., 131, 421-429 (2011)
4. V. Sahar, B. Arijit, P.J. Byrne, A case analysis of a sustainable food supply chain distribution system-A multi-objective approach, Int. J. of Pro. Eco., 152, 71-87 (2014)

5. C.S. Revelle, G. Laporte, The plant location problem: New models and research prospects, Ope. Res., 44, 864-874 (1996)

6. B.H. Sabri, A. Beamon, A multi-objective approach to simultaneous strategic and operational planning in supply chain design, Omega, 28, 581-598 (2011)

7. F.T.S. Chan, S.H. Chung, S. Wadhwa, A hybrid genetic algorithm for production and distribution, Omega, 33, 345-355 (2005)

8. C.L. Chen, W.C. Lee, Multi-objective optimization of multi-echelon supply chain networks, with uncertain product demands and prices, Com. \& Chem. Eng., 28, 1131-1144 (2004)

9. F. Altiparmak, M. Gen, L. Lin, T. Paksoy, A genetic algorithm approach for multiobjective optimization of supply chain Networks, Com. \& Ind. Eng., 51, 197-216 (2006)

10. P. Schütz, A. Tomasgard, S. Ahmed, Supply chain design under uncertainty using sample average approximation and dual decomposition, Eur. J. of Ope.Res., 199, 409-419 (2006)

11. B.L. Shankar, S. Basavarajappa, C.H. Jason, S. Chen, Location and allocation decisions for multi-echelon supply chain network - A multi-objective evolutionary approach, Exp. Sys. with App., 40, 551-562 (2013)

12. H. W. Stoll, Product Design Methods and Practices, (New York-Basel: Marcel dekker, Int, 1999)

13. A. Mohammed, Q. Wang, Integrity of an RFIDenabled HMSC network, Proceedings of the $3^{\text {rd }}$ ICDEIS, China, 79-86 (2015)

14. A. Ahmadia, H. Moghimib, A.E. Nezhade, G. Vassilios, M. Sharafd, Multi-objective economic emission dispatch considering combined heat and power by normal boundary intersection method, Ele. Pow. Sys. Res., 129, 32-43 (2015)

15. R.G. Pandu, Multi-Objective Optimization: Techniques And Applications, In Chem. Eng. (Adv. in Pro. Sys. Eng.), Singapore: WSP (Dec 2008)

16. HMC, UK. Available: http://www.halalhmc.org/testdemo.htm, (accessed October 26, 2014) 